\title{
BEHAVIOR-BASED CONTROL FOR SEMI-AUTONOMOUS ROBOTS *
}

\author{
João Sequeira ${ }^{1}$ M. Isabel Ribeiro ${ }^{1}$ \\ Instituto Superior Técnico/Institute for Systems and \\ Robotics, Av Rovisco Pais 1, 1049-001 Lisboa, Portugal
}

\begin{abstract}
This paper describes a hybrid architecture for robot control supported on basic properties of Hilbert spaces.

The proposed architecture is in the class of behavior based paradigms, with the control objectives specified as goal sets to be reached by the robot. This characteristic is well adapted to the control of semi-autonomous robots as tasks can be loosely specified by external operators using goal regions in the workspace where to steer the robots.

Simulation results on the control of a car-like robot are presented.
\end{abstract}

Keywords: Semi-autonomous robots, Hybrid systems, Behavioral robotics

\section{INTRODUCTION}

Semi-autonomous robots have been gaining importance in the last few years after the slow increase in the use of standard autonomous robotics in practical applications. Despite the vast work in the field of autonomy, where multiple architectures have been proposed and a large number of functionalities identified, intelligent control architectures have still to evolve at the higher decision levels that are often required by full autonomy.

Applications such as surveillance in wide open areas, rescue missions in catastrophe scenarios and the maintenance of remote infrastructures are examples of socially/economically relevant applications where autonomous robots can be used. Current state of the art locomotion/manipulation technologies are able to remotely operate in most of these environments under guidance of an external operator. Relevant examples can be found

* This work was supported by the FCT project POSI/SRI/40999/2001 and Programa Operacional Sociedade de Informação (POSI) in the frame of QCA III.

1 \{jseq,mir\}@isr.ist.utl.pt in minning, sewers inspection and maintenance, in aerial surveillance and even in medicine with some remote surgical interventions. The interactions between robots and operators must account for the fast dynamics of real environments that require fast control actions to be taken either by the human operators or the robots. The teleoperation concept from the early days of robotics evolved to control paradigms in which (i) the human operator only interacts with the robot at sparse instants providing decision capabilities whenever that of the robot reaches its limit, and (ii) the interaction is specified in loose form, i.e., goals are specified up to some uncertainty.

The approach to control of semi-autonomous robots considered in this paper has two distinctive features: (i) there is not enough information on the environment so that a specific goal position for the robot can be defined, or (ii) only a motion trend, or behavior, is specified in the form of a goal region to be reached by the robot. Therefore, instead of specifying a goal configuration or a trajectory to be followed by the robot, this paper addresses the situation where a set of goal configurations 
is admissible from a task execution perspective. These goal configurations induce the aforementioned motion trend and hence the behavior of the robot.

The paper is organised as follows. Section 2 describes the control algorithm supported on basic concepts from Hilbert spaces. Section 3 presents simulation results for a car-like robot (simulation experiments for unicycle robots, operating isolated and in a team, were presented in (Sequeira and Ribeiro, 2003a)). Section 4 presents the conclusions and discusses research directions. Appendix A details the basic tools on the geometry of Hilbert spaces that are used in the paper. Appendix B introduces the stability theorem of the generalized Lyapunov direct method used in Section 2 to develop arguments on the conditions for the successful execution of a mission by the robot.

\section{BEHAVIORAL CONTROL ON HILBERT SPACES}

For the purpose of this paper, robots are modelled by differential inclusions of the form

$$
\dot{q} \in f(q, U)
$$

where $q \in Q$ stands for the $C$-space variable and $U$ is a compact control space.

Users are assumed to interact with the robot by specifying goal regions to be reached by the robot in a behavioral space ${ }^{2}$. This form of interaction is close to what humans do when defining a task to be executed. For example, when driving a car a human driver is constantly selecting regions in its visual space where to steer the car; in some sense, this corresponds to the generation of a task achieving behavior. Similarly, an external user can control a robot by specifying goal regions to be reached this being a generalization of the standard trajectory following control problem.

From a behavioral perspective, a region of interest is a subset $K \subset Q$ that can either be specified by an external user with a given purpose or be defined by the robot control system. Such regions can be easily extracted from sensor data, e.g., a region of a specific brightness or color in an video image, or a region with particular range values in a range image.

Once a region of interest $K$ is defined, the set of motion directions at the current configuration $q$ that point to $K$ is defined as $\Delta_{K}(q)=K-q$. The set $\Delta_{K}(q)$ does not account for any motion constraints imposed by the robot kinematics. For a holonomic robot, it suffices to use any control

\footnotetext{
2 A space where the control objectives are defined.
}

such that $F_{K}(q) \equiv f(q, U) \cap \Delta_{K}(q) \neq \emptyset$ to have the robot reaching the goal set $K$. For nonholonomic robots $F_{K}(q)$ is often empty, as the motion is constrained by the kinematics, and hence maneuvering by the robot is required. Furthermore, the set $K$ can be specified in regions such that complex maneuvering is necessary.

Sufficient conditions for the sets $f(q, U)$ and $\Delta_{K}(q)$ to converge to each other whenever their intersection is empty are presented in the following Lemma (see Appendix A) that is the core of the control algorithm proposed in this paper.

Lemma 1. (Point-to-set convergence).

Let $Q$ be a Hilbert space, $K \subset Q$ a convex set, $q \in Q \backslash K$ and define

$$
\lambda=\left\langle\dot{q}, T_{K}^{\star}(q)\right\rangle,
$$

where $T_{K}^{\star}(q)$ stands for the conjugate cone to $K$ at the nearest point to $q$ belonging to $K$.

If the controls are chosen such that,

$$
\exists_{T>0}: \forall_{t>T}, \lambda>0
$$

then $q \rightarrow K$ as $t \rightarrow \infty$.

Roughly, if the projection of some admissible velocity vector, $\dot{q}$, over any direction leading to the goal set $T_{K}^{\star}(q)$ is positive, then this velocity vector leads to a decrease in the distance between the robot and the goal set. Although Lemma 1 was tailored to situations when $F_{K}(q)=\emptyset$ it is clear that controls in $F_{K}(q) \neq \emptyset$ lead to $\lambda \geq 0$ as the distance between the robot and the goal set also diminishes.

Lemma 1 suggests a partition for the control set in three disjoint regions, $U=U_{1} \cup U_{2} \cup U_{3}$, where each of the $U_{i}$ contains, respectively, the controls leading to $\lambda>0, \lambda=0$ and $\lambda<0$. Controls in $U_{1}$ guarantee that the robot approaches its goal. Controls in $U_{3}$ do not guarantee the decrease in the distance between the robot and its goal. Nevertheless, they may still be useful to maneuver the robot to a point that simplifies the reaching of the goal. Controls in $U_{2}$ guarantee that the distance between the robot and its goal does not increase and, depending on the specific problem, may even decrease. Table 1 summarizes the control algorithm.

To account for the conditions in Lemma 1, the algorithm starts by extracting a convex goal set $K$ out of the data provided by the mission specifications and/or by the onboard sensors. Extracting a convex set out of an acquired raw $\Delta_{K}$ can be made using computational geometry techniques (see, for example, (Preparata and Shamos, 1985)).

Choosing a control that maximizes $\lambda$ corresponds to the maximization of the rate at which the 


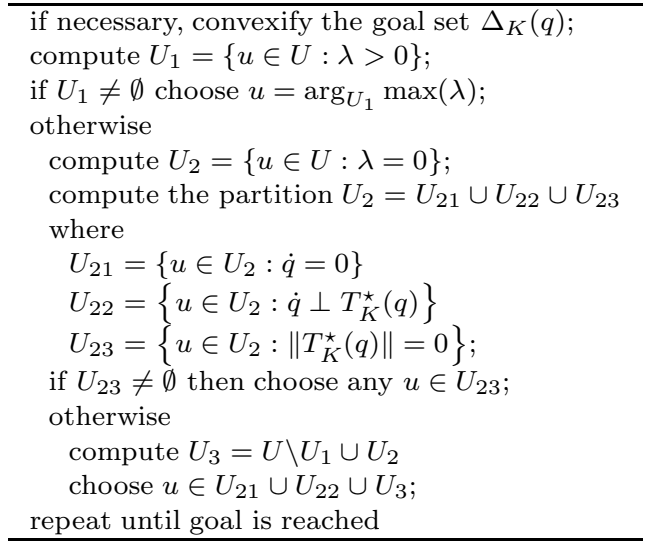

Table 1. Robot control algorithm

distance between the robot and the goal $K$ decreases. Controls in $U_{21}$ do not cause any progress in the motion. Unless the robot reached the goal or the robot has to be stopped during maneuvering, these controls must be avoided. Under controls in $U_{22}$ the robot moves in such a way that the distance to $K$ remains unchanged. For example, if $K$ is a disc in a $2 \mathrm{D}$ configuration space, controls in $U_{2}$ are those that steer the robot around the disc, keeping the distance between the robot and the disc. Controls in $U_{23}$ indicate a null distance between the robot and the goal set and hence are preferable among those in $U_{21} \cup U_{22} \cup U_{3}$. In some situations where $U_{1}=\emptyset$ it may be necessary to use controls in $U_{3}$ (see the first experiment in Section 3).

The algorithm in Table 1 has no deadlocks as each state has an output transition. However, the controls in $U_{21}, U_{22}$ and $U_{3}$ are defined after motion strategies specific to the robot and/or the mission assigned. If not properly chosen these may lead to a livelock situation in which the robot endlessly repeats a sequence of maneuvers ever reaching the goal.

A robot modelled by (1) and controlled by the algorithm in Table 1 is described by

$$
\dot{q} \in\left\{\begin{array}{l}
f\left(q, U_{1}\right) \text { if } \lambda>0 \\
f\left(q, U_{21}\right) \text { if } \dot{q}=0 \\
f\left(q, U_{22}\right) \text { if } \dot{q} \perp T_{K}^{\star}(q) \\
f\left(q, U_{23}\right) \text { if }\left\|T_{K}^{\star}(q)\right\|=0 \\
f\left(q, U_{3}\right) \text { if } \lambda<0
\end{array}\right.
$$

where $\left\|T_{K}^{\star}(q)\right\|=0$ indicates that the robot is at the boundary of $K$, i.e., the distance to $K$ measured from the current configuration, $q$, along a direction in $T_{K}^{\star}(q)$ is zero.

A sufficient condition for a mission to end successfully is that $U_{1} \cup U_{23} \neq \emptyset$. This means that either $U_{1} \neq \emptyset$ and hence the robot is at a configuration where it can approach $K$ or $U_{23} \neq \emptyset$ and hence the robot has reached a point in the boundary of $K$, $\mathrm{Bd}(K)$. When $q \rightarrow \mathrm{Bd}(K)$ using motion directions in $F_{K}(q),\left\|\Delta_{K}(q)\right\| \rightarrow 0$, meaning that a point in the kernel of $F_{K}(q)$ is reached. The motion directions generating controls in $U_{23}$ though may not stop the robot still represent a successful mission as the robot reaches a point in the boundary of $K$. Nevertheless, without lack of generality, $f\left(q, U_{23}\right)$ can be extended by

$f\left(q, U_{23}\right)=\left\{\begin{array}{l}\mathbf{0} \text { if } \forall u \in U_{23}, f(q, u) \ni \operatorname{Ker}(f(q, U)) \\ f\left(q, U_{23_{s}}\right) \text { otherwise }\end{array}\right.$

where $U_{23_{s}} \subset U_{23}$ is the set of controls such that $\forall u \in U_{23_{s}}, f(q, u) \in \operatorname{Ker}(f(q, U))$. In practical terms, this extension corresponds to force the robot to stop whenever it touches $K$ (for the sake of simplicity it is assumed that the kinematics allow sudden stops and dynamics side effects are ignored). A similar extension can be made for $f\left(q, U_{1}\right)$. The set of equilibria defined by $\operatorname{Ker}\left(F_{K}(q)\right)$ along with those introduced by the extensions of $f\left(q, U_{1}\right)$ and $f\left(q, U_{23}\right)$ define the conditions for the success of a mission.

The kernels of the remaining terms in the righthand side of (3) define alternative equilibria that correspond to situations where the robot stops before reaching $K$ and hence must be avoided.

Following the conditions in the Lyapunov direct method (see Appendix B for a brief presentation and (Smirnov, 2002; Aubin and Cellina, 1984) for further details) to hold for a system in the form (3), the upper Dini derivative of a Lyapunov function for (3) must be bounded by a negative function for stable equilibria.

Given that $\lambda>0$ is a sufficient condition for the mission to be successfull, if there is a sequence of controls corresponding to a sequence of switchings among the set valued maps in the righthand side of (3) resulting in a successful mission, then $\lambda^{2}$ is a Lyapunov function for this system.

The computation of the Dini derivative for $\lambda^{2}$, $D^{+} \lambda^{2}$, depends on the particular robot. The existence of a Lyapunov function implies that the hybrid system (3) reaches an equilibrium and hence, for the adequate sequence of controls (which is problem dependent), any mission can be successfully completed. Under the conditions of the Lyapunov theorem, it suffices to prove that $D^{+} \lambda^{2}$ is bounded to ensure that $\lambda^{2}$ is a Lyapunov function. By construction, $\lambda$ is bounded as it is piecewise smooth and exhibits only discontinuities of the first order (see the plots in Section 3). Therefore it is lower semi-continuous, and hence of $\lambda^{2}$, which means that it is upper bounded by a negative function, (Bacciotti et al., 2000).

The practical interpretation of the Lyapunov theorem is well known in hybrid systems theory, namely, the envelop of the Lyapunov function is strictly decreasing along the trajectory of the system towards the stable equilibria (see for instance (Lygeros, 1999)). 


\section{SIMULATION RESULTS}

This section presents simulation experiments on the control of a car-like robot (see Figure 1) to illustrate the results in Section 2. These experiments aim at demonstrating the feasibility of the hybrid control scheme in Table 1. The robot mission is to reach a goal set region defined by a ball of radius 0.1 centered at a point $q_{K}$. No obstacles are considered. However, it is worth to note that these can easily be accounted for by proper definition of the goal sets to be reached.

Two classes of experiments are presented: (i) the control is performed in the full $C$-space and (ii) only the $x y$ workspace is used. The ball assumption for the goal set $K$ simplifies the computation of $T_{K}^{\star}$ to

$$
T_{K}^{\star}(q)=-\alpha\left(\pi_{K}(q)-q\right) /\left\|\pi_{K}(q)-q\right\|
$$

where $\pi_{K}(q)=q_{K}+0.1\left(q-q_{K}\right) /\left\|q-q_{K}\right\|$ stands for the best approximation projection of $q$ onto $K$ (see Appendix A) and $\alpha>0$ is an arbitrary constant.

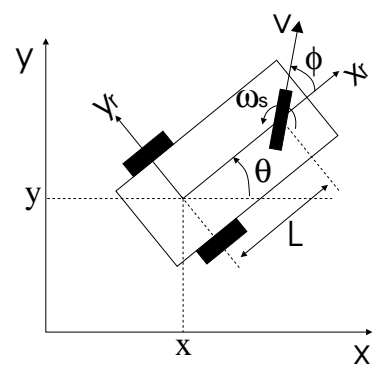

$$
\dot{q}=\left[\begin{array}{c}
\dot{x} \\
\dot{y} \\
\dot{\theta} \\
\dot{\phi}
\end{array}\right]=\left[\begin{array}{cc}
\cos (\theta) \cos (\phi) & 0 \\
\sin (\theta) \cos (\phi) & 0 \\
\frac{1}{L} \sin (\phi) & 0 \\
0 & 1
\end{array}\right]\left[\begin{array}{c}
v \\
\omega_{s}
\end{array}\right]
$$

Figure 1. The car-like mobile robot

The computation of the partition for $U$ in Table 1 for a generic robot may be computationally intensive. Exhaustive search techniques can be used to evaluate the projection map and avoid solving inverse dynamics problems, e.g., computing $u$ from $\dot{q}$, if the control space is discretized into a small number of admissible values. At each instant, a compact set of controls, $V \times \Omega_{s}=[-1: 0.5: 1] \times$ $[-1: 0.1: 1]$, is used to search for $U_{1}$. The steering wheel angle is constrained to $-\pi / 2 \leq \phi \leq \pi / 2$. In all the experiments, whenever $\lambda=0$ the controls are kept as before.

Figure 2 illustrates the results obtained when the algorithm is applied in the configuration space $[x, y, \theta, \phi]$. The robot starts from configuration $[0,0,0,0]$ and the goal region is set to a ball of 0.1 radius centered at $q=[1,1,0,0]$. To reach position $[x, y]=[1,1]$ with null orientation and subject to the considered compact control set requires a complex (with numerous maneuvers) trajectory. The first part is smooth and corresponds to the minimization of the distance along the $x$ coordinate. After a re-orientation maneuver around $[x, y]=[1,0]$, the robot proceeds to minimize the $y$ coordinate. This maneuver can be identified in the $\lambda$ plot around the instant of the first 0 crossing. The final approach to the goal set is made through a number of complex maneuvers, with the robot moving back and forth to reach a suitable orientation. The $\lambda$ plot shows that for the most of the time controls in $U_{3}$ are used. These are mainly needed for the maneuvering shown in the $x y$ plot. After a brief initial period where $\lambda$ decreases to 0 (corresponding to the initial horizontal part of the $x y$ trajectory) the system enters a heavy maneuvering phase. The final part of the mission corresponds again to a decrease of $\lambda$ to 0 . From the plot it is clear that, after the spike near $100 \mathrm{~s}$, the envelop of $\lambda^{2}$ is strictly decreasing, as expected from the Lyapunov theorem.

Figure 3 shows the results when the behavioral space is restricted to $[x, y]$. In this case the control variable is chosen as $u=\cos (\phi) v$. Given the kinematics of the robot, a natural objective when choosing $\omega_{s}$ is to require that $\theta+\phi$ tends to the direction of the goal set. Thus, $\omega_{s}$ is chosen to approach this direction. No bounds were assumed for the controls outside $U_{1}$. The $\lambda$ plot exhibits a smooth behavior decreasing to 0 , indicating that no harsh maneuvers were used.

Figure 4 shows the results when controls outside $U_{1}$ are bounded, with $|v| \leq 10$ and $\left|\omega_{s}\right| \leq 13$. Although the $x y$ trajectory is almost identical to the one in Figure 3, the behavior of the control system is completely different. During the first part of the experiment (visible as the horizontal region in the distance plot) the robot slowly changes the orientation of the steering wheel after which it proceeds towards the goal set. The initial horizontal trajectory for $\lambda$ is caused by the reorientation of the steering wheel. Around $4.4 \mathrm{~s}$ the plot shows a spike that indicates the beginning of the motion of the robot in the $x y$ plane. Clearly, the behavior of the $\lambda^{2}$ is also upper bounded by a decreasing curve, hence verifying the stability of the equilibrium state corresponding to the end of the mission.

\section{CONCLUSIONS}

The paper described the basics of a hybrid controller supported on basic concepts from Hilbert spaces. Standard results from generalized calculus were used to demonstrate the existence of a Lyapunov function such that, under general as- 

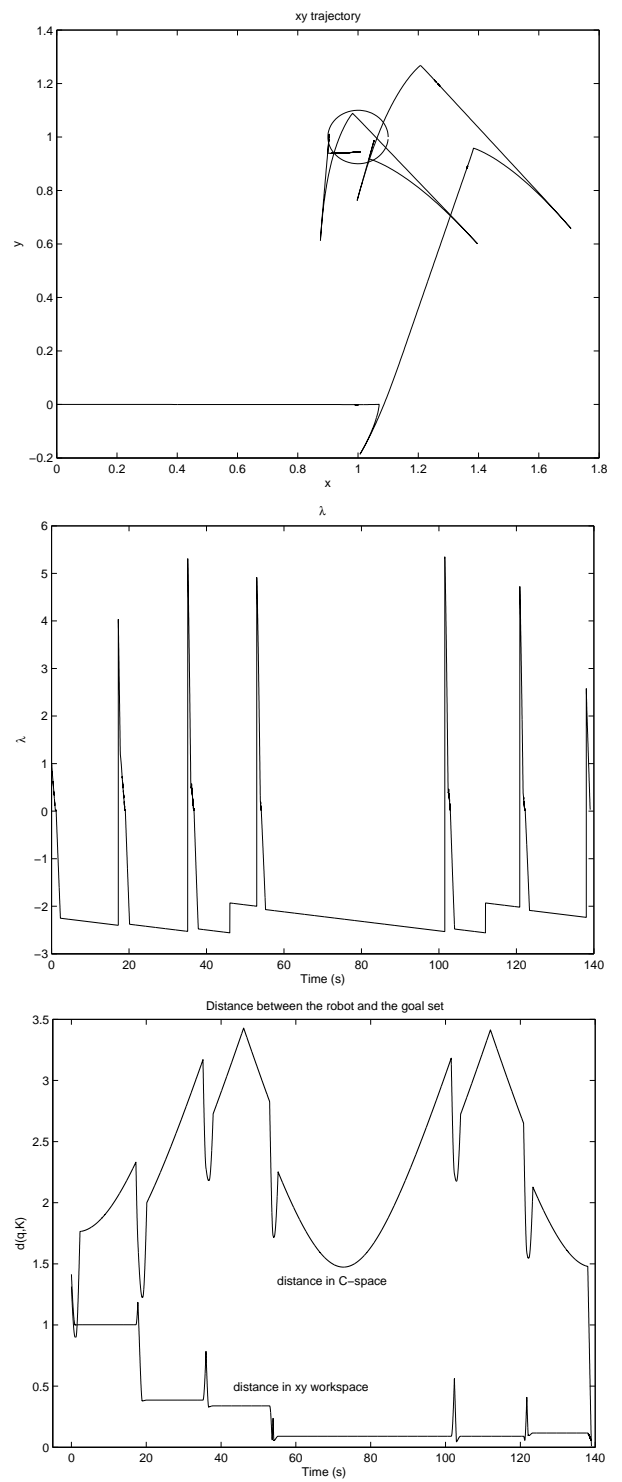

Figure 2. Car control in the $(x, y, \theta, \phi) C$-space sumptions on the robot model, the hybrid control structure yields a successful mission.

The simulation experiments show that the specification of a task as a goal region for the robot to reach can lead to reasonable robot trajectories. This represents a promising approach to alternative control strategies for semi-autonomous robots under the premises referred in Section 1.

Further research on both the analysis and the synthesis problems related with this work is necessary, namely in what concerns design requirements for the motion strategies to be applied when $\lambda \leq 0$.

\section{Appendix A. BASIS TOOLS ON THE GEOMETRY OF HILBERT SPACES}

Definition 1. (Distance between subsets). Let $A$ and $B$ be two subsets of a normed space. The distance between $A$ and $B$ is defined as
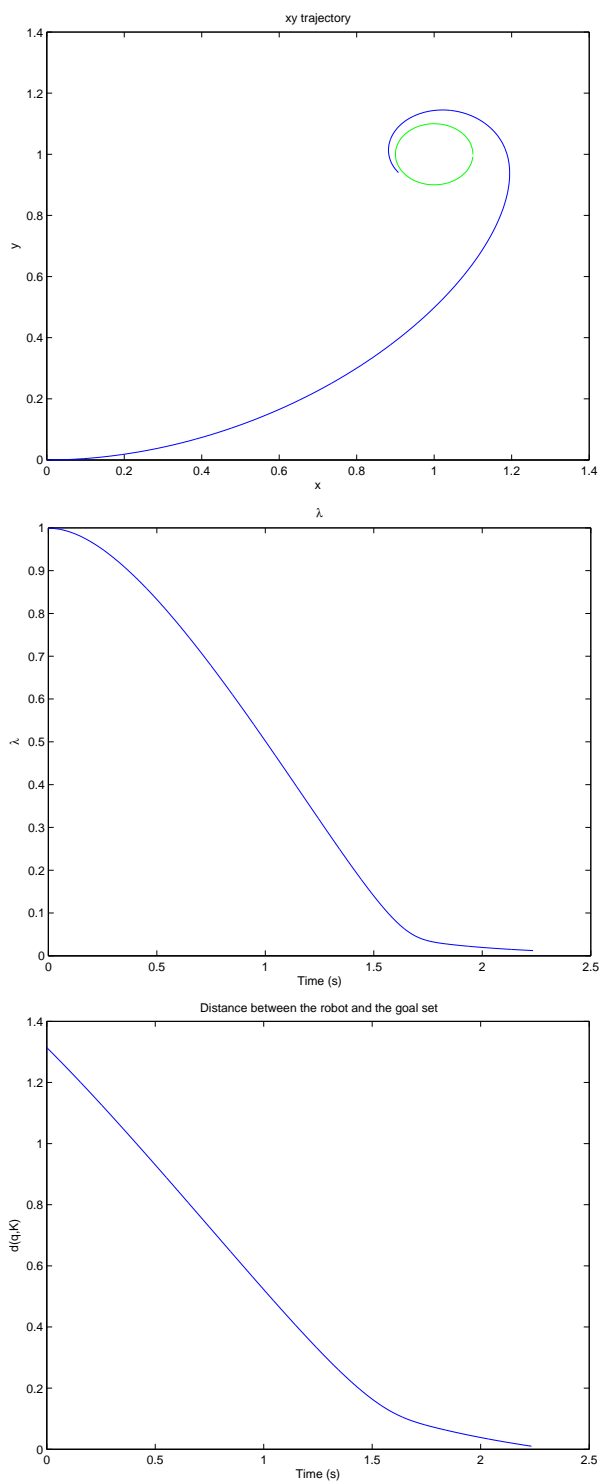

Figure 3. Car control in the $(x, y)$ workspace

$$
d(A, B)=\min _{x \in A, y \in B}\|x-y\|
$$

where $\|\cdot\|$ stands for a norm. Although commonly referred as a distance, (A.1) is not a metric. The distance between a point $x_{a} \in A$ and a set $B$ is defined by making $A$ a singleton.

The following theorem defines a projection map of a point onto a set. See (Aubin and Cellina, 1984) for a demonstration.

Theorem 1. (Best Approximation Theorem). Let $K$ be a closed convex subset of a Hilbert space, $X$. There is a map $\pi: X \mapsto K$ and a unique element $\pi_{K}(x)$ verifying

$$
\forall_{x \in X}, \quad\left\|x-\pi_{K}(x)\right\|=\min _{y \in K}\|x-y\|
$$

The map $\pi: X \mapsto K$ projecting $X$ into the goal set $K$ is called a best approximation projector (b.a.p.) whenever the following two conditions are 

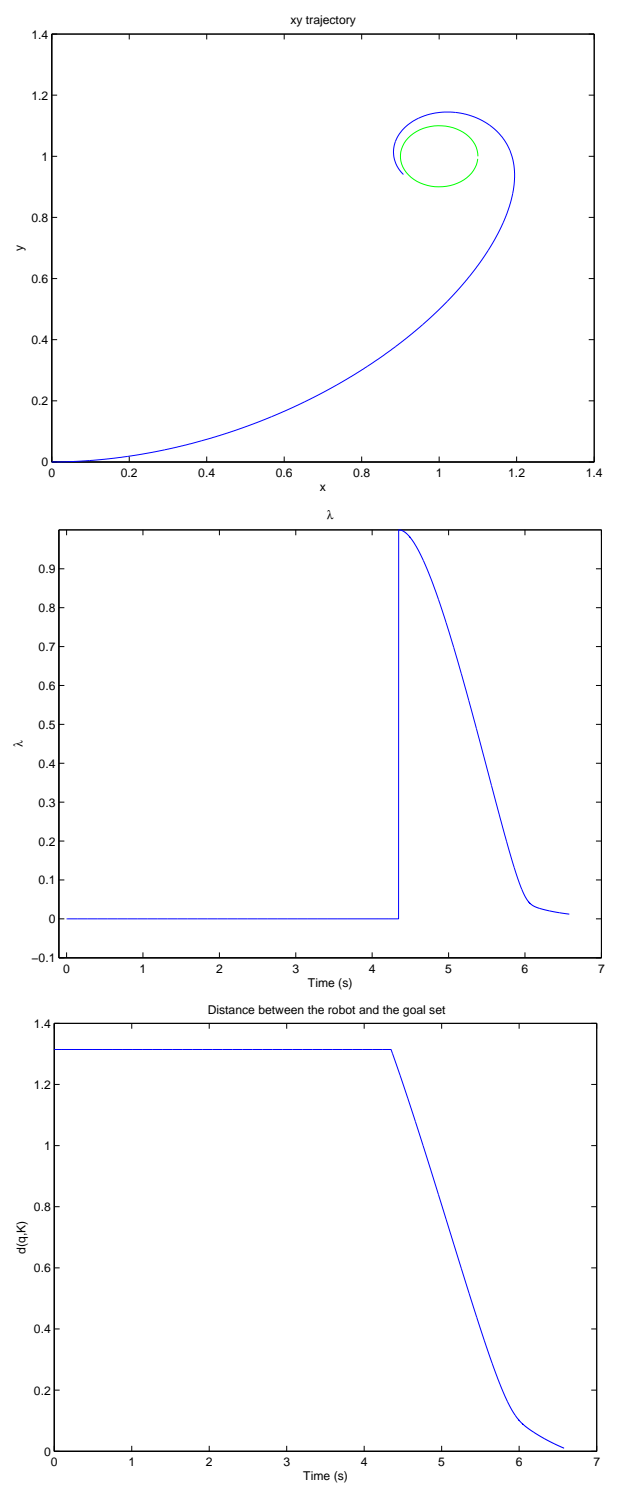

Figure 4. Car control in the $(x, y)$ workspace Both controls bounded

verified

$$
\begin{array}{ll}
\left\|\pi_{K}(x)-\pi_{K}(y)\right\| \leq\|x-y\| & \text { non-expansivity } \\
\left\langle\pi_{K}(x)-\pi_{K}(y), x-y\right\rangle \geq 0 & \text { monotonicity }
\end{array}
$$

with $\langle\cdot, \cdot\rangle$ standing for an inner product and $\|\cdot\|$ for the corresponding induced norm.

The convergence between the set of admissible velocities and the set of goal velocities is a generalization of the convergence between the current velocity and the set of goal velocities. The solution of this last problem implies a solution of the former.

Lemma 2. (Point-to-set convergence). Let $X$ be a Hilbert space, $K \subset X$ a convex set, $x \in X \backslash K$ and $\pi_{K}(x)$ a b.a.p. of a point $x$ onto $K$. Furthermore, let $x_{n}=x\left(t_{n}\right)$ be a subsequence of a trajectory $x(t)$, outside $K$, and define

$$
\lambda=\left\langle x_{n+1}-x_{n}, \pi_{K}\left(x_{n+1}\right)-x_{n+1}\right\rangle,
$$

such that, $\exists_{T>0}: \forall_{t_{n}>T}, \lambda>0$. Then $d\left(x_{n}, K\right) \rightarrow 0$ as $n \rightarrow \infty$.

When $x_{n+1} \rightarrow x_{n}$ the condition in Lemma 2 can be formulated in continuous time, as

$$
\left\langle\dot{x}, T_{K}^{\star}(x)\right\rangle>0
$$

where $T_{K}^{\star}(x)$ represents the conjugate cone to $K$ at $\pi_{K}(x)$, i.e., the set of vectors orthogonal to the set tangent to the border of $K$ at $\pi_{K}(x)$.

See (Sequeira and Ribeiro, 2003b) for a demonstration.

\section{Appendix B. LYAPUNOV DIRECT METHOD}

This section presents the basics on Lyapunov direct method (see for instance (Smirnov, 2002) for details).

The upper Dini derivative of a function $V(q)$ at vector $v$ is defined by

$$
D^{+} V(q)(v)=\lim _{h \rightarrow 0} \sup _{v^{\prime} \rightarrow v} \frac{V\left(q+h v^{\prime}\right)-V(q)}{h}
$$

Consider the system $\dot{q} \in F(q)$, where $F: \mathbb{R}^{n} \mapsto \mathbb{R}^{n}$ and $0 \in \operatorname{Ker}(F(q))$.

Theorem 2. (Lyapunov stability theorem). Assume that there exist $\eta>0$, a positive definite function $V: \mathbb{R}^{n} \mapsto \mathbb{R}$ and a negative definite function $W: \mathbb{R}^{n} \mapsto \mathbb{R}$ such that

$$
\forall v \in F(q),|q| \leq \eta, \quad D^{+} V(q)(v) \leq W(q)
$$

Then the equilibrium position $q=0$ is asymptotically stable.

\section{REFERENCES}

Aubin, J.P. and A. Cellina (1984). Differential Inclusions. Springer-Verlag.

Bacciotti, A., F. Ceragioli and L. Mazzi (2000). Differential inclusions and monotonicity conditions for nonsmooth lyapunov functions. Set Valued Analysis 8, 299-309.

Lygeros, J. (1999). Hybrid systems: Modelling analysis and control. Teaching/ee291E.html.

Preparata, F. and M. Shamos (1985). Computational Geometry: An Introduction. SpringerVerlag.

Sequeira, J. and M. I. Ribeiro (2003a). A geometric approach to single and multiple robot control. In: Proceedings of the 7th IFAC Symposium on Robot Control, Syroco 2003.

Sequeira, J. and M. I. Ribeiro (2003b). Geometric control of robot teams. Technical Report RT602-03. Institute for Systems and Robotics.

Smirnov, G. (2002). Introduction to the Theory of Differential Inclusions. Vol. 41 of Graduate Studies in Mathematics. American Mathematical Society. 